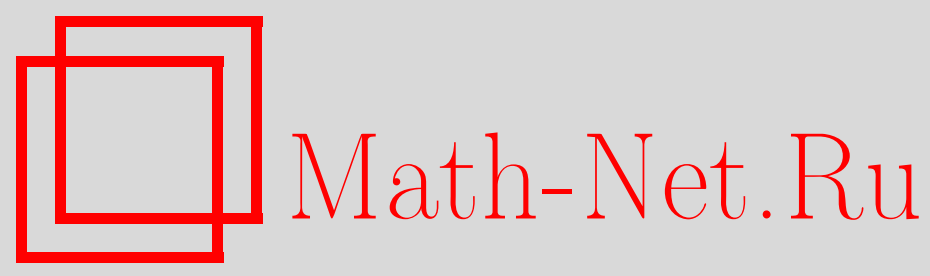

В. В. Кабанов, О графах без корон с регулярными $\mu$ подграфами, Матем. заметки, 2000, том 67, выпуск 6, 874881

DOI: https://doi.org/10.4213/mzm906

Использование Общероссийского математического портала Math-Net.Ru подразумевает, что вы прочитали и согласны с пользовательским соглашением http://www . mathnet.ru/rus/agreement

Параметры загрузки:

IP: 3.93 .64 .190

26 апреля 2023 г., 16:29:47

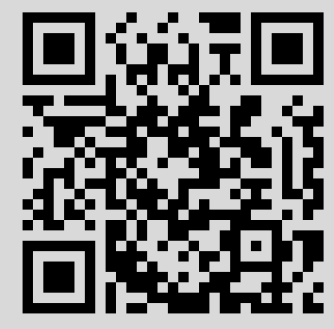




\title{
О ГРАФАХ БЕЗ КОРОН С РЕГУЛЯРНЫМИ $\mu$-ПОДГРАФАМИ
}

\author{
В.В. Кабанов
}

Назовем $m$-короной полный трехдольный граф̆ $K_{1,1, m}$ с долями порядков $1,1, m$, а $m$-лапой-полный двудольный граф $K_{1, m}$ с долями порядков $1, m$ при $m \geqslant 3$. Вершину $а$ графа $Г$ назовем слабо редуцированной, если подграф̆ $\left\{x \in \Gamma \mid a^{\perp}=x^{\perp}\right\}$ состоит из единственной вершины. Граф Г называется слабо редуцированным, если все его вершины слабо редуцированы. В данной работе классифицированы связные слабо редуцированные графы без 3-корон, в которых все $\mu$-подграфы являются регулярными графами одной ненулевой валентности. В частности, обобщается характеризация графов Грассмана и Джонсона, полученная Нуматой, и характеризация связных редуцированных графов без 3-лап, полученная Махневым.

Библиографиял: 11 названий.

Введение. Рассматриваются только конечные неориентированныеграфы без петель и кратных ребер. Мы называем подграфом графа Г граф на подмножестве вершин из $\Gamma$, у которого вершины смежны тогда и только тогда, когда они смежны в Г. Обычно такой подграф назьвают порожденным. Для вершины $a$ графа $\Gamma$ через $\Gamma_{i}(a)$ обозначим подграф на множестве всех вершин, которые находятся на расстоянии $i$ от вершины $a$. Через $\Gamma(a)$ обозначим $\Gamma_{1}(a)$. Если граф $\Gamma$ зафиксирован, то $\Gamma(a)$ обозначим через $[a]$. Этот подграф назовем окрестностью вершины $a$. Подграф на множестве $[a] \cup\{a\}$ обозначим через $a^{\perp}$. Для подграфа $\Delta$ из $\Gamma$ обозначим через $[a]_{\Delta}$ окрестность $a$ в $\Delta$, а $a^{\perp} \cap \Delta$ через $a_{\Delta}^{\perp}$. Если $a$ и $b-$ вершины из графа $Г$ и расстояние между ними в $Г$ равно 2, то подграф на множестве всех вершин, которые смежны с ними обеими, будем назьвать $\mu$-подграфом. Пусть $\mathscr{F}-$ класс графов. Граф Г назьвается локально $\mathscr{F}$-графом, если окрестность каждой вершины из Г принадлежит $\mathscr{F}$. Если все графы класса $\mathscr{F}$ изоморфны одному графу $G$, то локально $\mathscr{F}$-граф будем называть локально $G$-графом. Кликой (кокликой) мы называем полньй (вполне несвязный) подграф. Через $K_{m_{1}, m_{2}, \ldots, m_{t}}$ обозначим полньй многодольный граф с $t$ долями порядка $m_{1}, m_{2}, \ldots, m_{t}$. Если $m_{1}=m_{2}=\cdots=m_{t}$, то $K_{m_{1}, m_{2}, \ldots, m_{t}}$ обозначим $K_{t \times m}$. Граф $K_{1, m}$ назовем $m$-лапой, а граф $K_{1,1, m}-m$-короной, если $m \geqslant 3$.

В статье [1] Броувером и Нуматой, описаны все (в том числе и бесконечные) графы без 3-лап с несвязными $\mu$-подграфами. Используя этот результат и свою классификацию реберно регулярных графов без 3-лап [2], Нумата в работе [3] получил характеризацию графов Грассмана и Джонсона как графов локально без 3-лап, в которых все

Работа выполнена при финансовой поддержке Российского фонда фундаментальных исследований, грант № 99-01-00462. 
$\mu$-подграфы являются изоморфными реберно регулярными графами диаметра 2 . Заметим, что из последнего условия на все $\mu$-подграфы графа Г непосредственно следует реберная регулярность самого Г.

Наша цель получить классификацию локально без 3-лап графов с более слабым условием на $\mu$-подграфы, которое не влечет даже регулярности графа. Это стало возможно благодаря работам Махнева и автора [4]-[6], в которых получена классификация графов без 3-лап с равномощными $\mu$-подграфами. С помощью этой классификации в данной статье исследуется класс графов, в которых окрестности вершин не содержат 3-лап и все $\mu$-подграфы являются регулярньми графами валентности $\alpha$, где $\alpha>0$. В отличие от [3], мы не требуем даже равномощности всех $\mu$-подграфов. Поскольку наше условие на $\mu$-подграфы не влечет регулярности графа, то мы дополнительно накладьваем на граф условие слабой редуцированности.

Очевидно, что класс графов без 3-лап является подклассом класса графов локально без 3-лап. Соответствующая классификация графов без 3-лап была получена Махневым в [7]. Регулярньй граф назьвается редуцированным, если для любой вершины $a \in \Gamma$ множество $\left\{x \in \Gamma \mid a^{\perp}=x^{\perp}\right\}$ состоит из единственной вершины $a$. В статье [7] Махнев обобщает это понятие на класс всех графов. Он назьвает произвольньй граф $\Gamma$ редуцированным, если для любой вершины $a \in \Gamma$ множество $\left\{x \in \Gamma \mid a^{\perp} \subseteq x^{\perp}\right\}$ состоит из единственной вершины $a$. Легко видеть, что в классе регулярных графов оба определения эквивалентны. В [7] Махнев классифицировал редуцированные графы без 3 -лап с регулярными $\mu$-подграфами валентности $\alpha$, где $\alpha>0$. Мы покажем, что если Г является графом без 3 -лап с регулярными $\mu$-подграфами одной валентности $\alpha$, где $\alpha>0$ и для любой вершины $a$ из $Г$ множество $\left\{x \in \Gamma \mid a^{\perp}=x^{\perp}\right\}$ совпадает с $\{a\}$, то и множество $\left\{x \in \Gamma \mid a^{\perp} \subseteq x^{\perp}\right\}$ состоит из единственной вершины $a$. Таким образом несколько обобщается основной результат из [7].

Под кликовым расширением графа Г будем понимать граф, полученньй заменой каждой вершины $a$ из Г на полньй подграф $C(a)$, содержаший не менее одной вершины, причем вершины из различных клик $C(a)$ и $C(b)$ смежны тогда и только тогда, когда вершины $a$ и $b$ смежны в $\Gamma$. Кликовое расширение графа $Г$ будем назьвать $\beta$-расиирением, если подграф $C(a)$ содержит $\beta$ вершин для любой вершины $a \in \Gamma$.

ТЕОРема 1. Пусть Г-связный граф, который удовлетворяет следующим условия⿻

1) Г не содержит 3-лап;

2) Г содержит 3-коклику;

3) все $\mu$-подграфы из Г являются регулярными графами одной валентности $\alpha$ для некоторого числа $\alpha>0$;

4) для любой вершинь $a \in \Gamma$ множество $\left\{x \in \Gamma \mid a^{\perp}=x^{\perp}\right\}$ coстоит из единственной вериины $а$.

Тогда граф Г является треугольным графом $T(n)$ при $n \geqslant 6$, графом икосаэдра или графом Шлефли.

Заметим, что класс графов без 3-коклик или, что то же самое, класс графов дополнительных к графам без треугольников довольно широк и подвергался интенсивному исследованию многими авторами (см., например, монографию [8]).

ТЕОРема 2. Пусть Г-связный граф, который удовлетворяет следующим усло- 
виям:

1) окрестность любой вершины из Г не содержит 3-лап;

2) окрестность некоторой вершины из Г содерэсит 3-коклику;

3) все $\mu$-подграфы из Г являются регулярными графами одной валентности $\alpha$ для некоторого числа $\alpha>0$;

4) для любой вершины $a \in \Gamma$ множество $\left\{x \in \Gamma \mid a^{\perp}=x^{\perp}\right\}$ coстоит из единственной вершины $а$.

Тогда Г является локально $G$-графом, где $G$ один из следующих графов:

(a) $\beta$-расширение $m \times n$ графа при $\beta=\alpha / 2$ u $m \geqslant 3, n \geqslant 3$;

(b) треугольный граф $T(n)$ nри $n \geqslant 6$;

(c) граф Шлефли.

ТЕОРемА 3. Пусть Г - связный граф, который удовлетворяет условиям теоремы 2, и все $\mu$-подграфы из Г имеют диаметр 2.

Тогда Г является одним из следующих графов:

(a) граф Грассмана;

(b) граф Джсонсона или его частное;

(c) локально $T(n)$-граф при $n \geqslant 6$;

(d) граф Госсета $E_{7}(1)$.

Приведем определения основных понятий, которые используются в статье.

Треугольным. графом $T(n)$ называется реберный граф полного графа с $n$ вершинами. Граф на множестве пар $X \times Y$ называется $m \times n$ әрафом (решетчатым графом), если $|X|=m,|Y|=n$, а пары $\left(x_{1}, y_{1}\right)$ и $\left(x_{2}, y_{2}\right)$ смежны тогда и только тогда, когда $x_{1}=x_{2}$, $y_{1} \neq y_{2}$ или $x_{1} \neq x_{2}, y_{1}=y_{2}$. Граф Шлефли-это сильно регулярный граф с параметрами $(27,16,10,8)$, которьй является дополнительным к точечному графу обобщенного четырехугольника $G Q(2,4)$ (см., например, [8, п. 10.3.4].

Пусть $V$ является $n$-мерным векторным пространством над конечным полем $F$. $Г p a$ фом Грассмана для $m$-подпространств из $V$ назьвается граф $\left[\begin{array}{c}V \\ m\end{array}\right]$ с множеством всех подпространств размерности $m$ из $V$ в качестве множества вершин, в котором две вершины $A, B$ смежны тогда и только тогда, когда $\operatorname{dim}(A \cap B)=m-1$.

Если $X$ - конечное множество, то графом Джсонсона для $m$-подмножеств из $X$ назьвается граф на множестве $\left(\begin{array}{c}X \\ m\end{array}\right)$ всех $m$-элементных подмножеств из $X$, в котором две вершины $a, b$ смежны тогда и только тогда, когда $|a \cap b|=m-1$. Если множество $X$ состоит из $n$ элементов, то такой граф обозначается $J(n, m)$. Заметим, что граф $J(n, 2)$ совпадает с треугольным графом $T(n)$.

Пусть $\rho$ является разбиением множества вершин графа Г на непересекающиеся подмножества. Частным $\bar{\Gamma}=\Gamma / \rho$ для графа $\Gamma$ по разбиению $\rho$ называется граф на фактор-множестве по $\rho$ множества вершин $\Gamma$, в котором две вершины $\bar{a}, \bar{b}$ смежны, только если $a \neq b$ и граф $\Gamma$ содержит ребро, которое соединяет некоторую вершину из класса $\bar{a} \mathrm{c}$ некоторой вершиной из класса $\bar{b}$. Пусть $n=2 m$ и $\rho$ является разбиением множества вершин графа Джонсона $J(2 m, m)$ на двухэлементные классы, содержащие $m$-элементное подмножество и его дополнение в $X$. Частным графа Джснсона называется граф $J(2 m, m) / \rho$.

Графы Джонсона и частные графов Джонсона дают нам примеры локально $(n \times m)$ графов. Эти примеры не исчерпывают класс таких графов. В статье [9] построены дру- 
гие примеры и найдены все локально $(4 \times 4)$-графы. Там жеполучено описание локально $(n \times m)$-графов, в которых каждый $\mu$-подграф является объединением изолированных 4 -циклов. Характеризация локально $(n \times m)$-графов при $n=3$ получена Холлом в [10]. Проблема описания локально $(n \times m)$-графов для любых $n, m$ остается открытой.

Граф Г диаметра $d$ назьвается дистаниионно регулярным с параметрами $a_{i}, b_{i}, c_{i}$, $i=1, \ldots d$, если для любой пары вершин $x, y$ из $\Gamma$ таких, что $d(x, y)=i$, найдется в точности $c_{i}$ соседей для вершины $y$ в $\Gamma_{i-1}(x)$, найдется в точности $b_{i}$ соседей для вершины $y$ в $\Gamma_{i+1}(x)$ и $a_{i}=b_{0}-b_{i}-c_{i}$.

Ректографом называется связный граф без треугольников, в котором любой путь длины 2 лежит в единственном четырехугольнике. Известно [11], что если ректограф дистанционно регулярен и если он имеет валентность $k$, параметры $a_{2}(\Gamma)=0$ и $c_{3}(\Gamma)=3$, то каждая связная компонента графа $\Gamma_{2}$ (граф 2-путей) является локально треугольным графом валентности $k(k-1) / 2$. Если Г будет двудольным графом, то $\Gamma_{2}$ имеет две компоненты (так называемые половинные графы). Неймайер в [11] показал, что каждый связньй локально треугольный граф Г является половинным графом двудольного ректографа с $c_{3}(\Gamma)=3$ и любой $\mu$-подграф из Г будет объединением непересекающихся подграфов, изоморфных $T(4)$.

Граф Госсета $E_{7}(1)$ имеет 56 вершин, и окрестность каждой вершины является графом Шлефли (определение см., например, в [8, с. 103]).

2. Доказательство теоремы 1. Пусть Г-граф, который удовлетворяет условию теоремы 1 , и $\alpha$-валентность всех $\mu$-подграфов из $\Gamma, \alpha>0$.

ЛЕмма 2.1. Если в графе Г окрестность любой вериины не содержит 3-коклик, то для произвольной вериины $а \in \Gamma$ множество $\left\{x \in \Gamma \mid x^{\perp} \subseteq a^{\perp}\right\}$ состоит из единственной вериины $а$.

ДокАЗАТЕЛЬСТво. Пусть окрестности всех вершин из графа $Г$ не содержат 3-коклик, и для некоторой вершины $y \in \Gamma$ найдется вершина $x$ из $[y]$, такая что $x^{\perp} \subset y^{\perp}$.

Выберем среди таких пар вершин $(y, x)$ пары $(a, x)$ такие, что вершина $a$ имеет наибольшую валентность в графе $\Gamma$. Среди пар $(a, x)$ выберем пару $(a, b)$ такую, что вершина $b$ имеет наибольшую валентность в графе $\Gamma$. Заметим, что подграф $[a]-[b]$ является кликой, поскольку графф Г не содержит 3 -лап.

1. Покажем, что если вершина $x$ из $[b]-\{a\}$ смежна с некоторой вершиной вне $a^{\perp}$, то она не смежна ни с одной вершиной из $[a]-b^{\perp}$. Действительно, пусть $y \in[x]-a^{\perp}$, $z \in([x] \cap[a])-b^{\perp}$. Тогда вершина $y$ смежна с $z$, иначе подграф на $\{x, b, y, z\}$ является 3 -лапой. С другой стороны, в $\mu$-подграфе $[y] \cap[b]$ вершина $x$ имеет валентность $\alpha$, а в $\mu$-подграфе $[y] \cap[a]$ вершина $x$ смежна еще с вершиной $z$ из $([y] \cap[a])-[b]$. Противоречие с условием на валентность $\mu$-подграфов.

2. Пусть $b^{\perp}$ является кликой. Тогда для вершины $c$ из $[a]-b^{\perp}$ по условию теоремы имеем $|[c] \cap[b]|=\alpha+1$. Пусть $d \in[c] \cap([b]-\{a\})$. Тогда в силу выбора вершины $b$ окрестность $[d]$ не содержится в $a^{\perp}$ и содержит вершину $c$ из $[a]-b^{\perp}$. Противоречие с пунктом 1.

Пусть $b^{\perp}$ не является кликой и $c, d$-пара несмежных вершин из $b^{\perp}$. Тогда $\left|[c] \cap[d] \cap a^{\perp}\right|$ $=\alpha+1$ и $[c] \cap[d] \cap a^{\perp}$ содержится в $b^{\perp}$. Значит, каждая вершина из $[a]-b^{\perp}$ смежна в точности с одной из вершин $c, d$.

3. Пусть $\left([a]-b^{\perp}\right) \cap[x]$ и $\left([a]-b^{\perp}\right) \cap[y]$ непустые подграфы для любой пары несмежных вершин $x, y$ из $[b]$. В этом случае по пункту 1 и в силу выбора вершины $b$ окрестность 
любой вершины из $b^{\perp}$ содержится в $a^{\perp}$. Поскольку граф Г связен, содержит 3-коклику и не содержит 3 -лап, то в $[a]-b^{\perp}$ найдется вершина $e$ смежная с некоторой вершиной $f$ вне $a^{\perp}$. Напомним, что $[a]-b^{\perp}$ является кликой. Не ограничивая общности рассуждений, можно считать, что е содержится в $\left([a]-b^{\perp}\right) \cap[c]$. Но тогда вершина $f$ смежна с любой вершиной $g$ из $\left([a]-b^{\perp}\right) \cap[d]$, иначе подграф на $e, c, f, g$ является 3 -лапой. Теперь такое же рассуждение для любой вершины из $\left([a]-b^{\perp}\right) \cap[d]$ показьвает, что $f$ смежна с любой вершиной из $[a]-b^{\perp}$. Но $f$ не смежна ни с одной вершиной из $b^{\perp}$. Значит, $[a] \cap[f]=[a]-b^{\perp}$ и поскольку $[a]-b^{\perp}$ является кликой, то $|[a] \cap[f]|=\alpha+1$. Далее, $[c] \cap[f] \subseteq[a] \cap[f]$ и, значит, $[c] \cap[f]=[a] \cap[f]$. Но тогда $\left([a]-b^{\perp}\right) \cap[d]-$ пустой подграф. Противоречие с выбором вершины $d$.

4. По пункту 3 для некоторой пары несмежных вершин $x, y$ из $b^{\perp}$ один из подграфов $\left([a]-b^{\perp}\right) \cap[x],\left([a]-b^{\perp}\right) \cap[y]$ является пустьм графом. Не ограничивая общности рассуждений, мы можем считать, что $\{x, y\}=\{c, d\}$ и $\left([a]-b^{\perp}\right) \cap[c]=[a]-b^{\perp}$, $\left([a]-b^{\perp}\right) \cap[d]=\varnothing$. Пусть $e \in[a]-b^{\perp}$. Тогда $\mu$-подграф $[d] \cap[e]$ содержит $\alpha+1$ вершину из $a^{\perp}$, причем все эти вершины содержатся в $b^{\perp}$ по выбору вершин $\{c, d\}$. Теперь $\mu$-подграф $[b] \cap[e]$ содержит вершину $a$, которая должна иметь в нем валентность $\alpha$. Однако вершина $a$ имеет в этом $\mu$-подграффе валентность не меньше $\alpha+1$, поскольку он содержит еще вершину $c$. Противоречие.

Теперь из доказанной леммы и теоремы Махнева [7] следует, что Г является треугольньг графом $T(n)$ при $n \geqslant 6$, графом Шлефли или графом икосаэдра.

3. Доказательство теоремы 2. Пусть Г-граф, который удовлетворяет условию теоремы 2 . Напомним, что все $\mu$-подграфы из $Г$ регулярны валентности $\alpha$ и $\alpha>0$.

Лемма 3.1. Окрестность любой вериинь из графа Г либо не содержит 3-коклик, либо является одним из следующих графов:

(1) $\beta$-расширение $(m \times n)$-графа при $\beta=\alpha / 2$ u $m \geqslant 3, n \geqslant 3$;

(2) $\beta$-расширение треугольного графа $T(n)$ при $\beta=\alpha / 4$ u $n \geqslant 6$;

(3) $\beta$-расширение графа Шлефли при $\beta=\alpha / 8$.

ДокАЗАТЕЛЬСТво. Пусть окрестность вершины $a$ из графа Г содержит 3-коклику. По условию теоремы все $\mu$-подграфы из Г являются регулярными графами валентности $\alpha$, где $\alpha>0$. Значит, для любой пары несмежных вершин из окрестности вершины $a$ их $\mu$-подграф пересекает $[a]$ по $\alpha$ вершинам. Таким образом, окрестность $[a]$ является графом без 3-лап с равномощными $\mu$-подграфами. Если все $\mu$-подграфы из [a] являются кликами, то подграф [a] удовлетворяет условию теоремы 1 из [4]. Поскольку [a] содержит 3-коклику, то по лемме 3.4 из [4] подграф [a] имеет диаметр больше 2. Пусть $b, c$ - пара вершин на расстоянии 3 друг от друга в $[a]$. Тогда вершина $a$ содержится в $\mu$-подграфе $[b] \cap[c]$ и является изолированной в $[b] \cap[c]$. Противоречие с условием $\alpha>0$. Значит, в $[a]$ есть пара несмежных вершин с некликовым $\mu$-подграфом. Но тогда по теореме 2 из [4] подграф [a] удовлетворяет заключению леммы. Лемма доказана.

Ядром подграфа $\Delta$, содержашего более одной вершины, мы будем называть подграф $K(\Delta)=\Delta^{\perp} \cap \Delta$. Ядром вершины а назьвается подграф $K(a)=\left\{x \in \Gamma \mid x^{\perp}=a^{\perp}\right\}$. Через $K_{\Delta}(a)$ обозначим ядро вершины $a$ в подграфе $\Delta$.

ЛЕмма 3.2. Окрестность любой вериины из Г содержит 3-коклику. 
ДокАЗАТЕЛЬСТво. По условию 2) теоремы окрестность некоторой вершины из Г содержит 3-коклику. Пусть окрестность вершины $a$ из графа $Г$ не содержит 3-коклик, а окрестность вершины $b$ содержит 3 -коклику. Поскольку $\Gamma$ является связным графом, то без потери общности рассуждений можно считать, что вершины $a$ и $b$ смежны. По лемме 3.1 окрестность вершины $b$ является одним из следующих графов: $\beta$-расширение $(m \times n)$-графа при $\beta=\alpha / 2, m \geqslant 3, n \geqslant 3 ; \beta$-расширение треугольного графа $T(n)$ при $\beta=\alpha / 4$, где $n \geqslant 6$; $\beta$-расширение графа Шлефли при $\beta=\alpha / 8$. Обозначим через $\Delta$ подграф $b^{\perp}$.

Пусть $[b]$ является $\beta$-расширением $(m \times n)$-графа при $\beta=\alpha / 2, m \geqslant 3, n \geqslant 3$. В этом случае $\left|K_{\Delta}(a)\right|=\beta$ и подграф $([a] \cap \Delta)-K_{\Delta}(a)$ будет объединением двух непересекающихся изолированных клик, скажем $A$ и $B$, содержащих $(m-1) \beta$ и $(n-1) \beta$ вершин соответственно. Поскольку $m \geqslant 3$ и $n \geqslant 3$, то подграфы $A$ и $B$ содержат не менее $\alpha$ вершин каждый. Пусть $c_{1} \in A, d_{1} \in B$. По условию 3$)$ теоремы в точности $\alpha$ вершин из $\mu$-подграфа $\left[c_{1}\right] \cap\left[d_{1}\right]$ содержится в $[a]$, причем $\beta$ из них в $K_{\Delta}(a)$ и $\beta$ - вне $\Delta$. Пусть $e_{1} \in\left(\left[c_{1}\right] \cap\left[d_{1}\right] \cap[a]\right)-K_{\Delta}(a)$. Аналогично, в точности $\alpha$ вершин из $\mu$-подграфра $[b] \cap\left[e_{1}\right]$ содержится в $[a]$. Поскольку $A$ и $B$ содержат не менее $\alpha$ вершин каждый, а вершины $c_{1} \in A, d_{1} \in B$ принадлежат $[b] \cap\left[e_{1}\right] \cap[a]$, то в $A$ и $B$ есть вершины не смежные с $e_{1}$. Пусть $e_{2} \in A-\left[e_{1}\right]$ и $e_{3} \in B-\left[e_{1}\right]$. Тогда $\left\{e_{1}, e_{2}, e_{3}\right\}$ является 3 -кокликой из $[a]$. Противоречие с выбором вершины $a$.

Пусть $[b]$ является $\beta$-расширением треугольного графа $T(n)$ при $\beta=\alpha / 2$, где $n \geqslant 6$. В этом случае $\left|K_{\Delta}(a)\right|=\beta$ и подграф $([a] \cap \Delta)-K_{\Delta}(a)$ будет $\beta$-расширением $((n-2) \times$ $2)$-графа для $n \geqslant 6$. Все вершины такого графа содержатся в двух непересекающихся кликах, скажем $A$ и $B$, содержащих по $(n-2) \beta$ вершин.

Пусть $c_{1}, d_{1}$ являются несмежными вершинами из $A \cup B$ и $c_{1} \in A, d_{1} \in B$. По условию 3$)$ теоремы в точности $\alpha$ вершин из $\mu$-подграффа $\left[c_{1}\right] \cap\left[d_{1}\right]$ содержится в $[a]$. В точности $\beta$ из них принадлежит $K_{\Delta}(a)$. Заметим, что подграф $\left(\left[c_{1}\right] \cap\left[d_{1}\right] \cap a^{\perp}\right)-K_{\Delta}(a)$ является объединением двух изолированных $\beta$-клик $\left[c_{1}\right] \cap B$ и $\left[d_{1}\right] \cap A$. Таким образом, $\beta$ вершин из $\left[c_{1}\right] \cap\left[d_{1}\right] \cap a^{\perp}$ не принадлежат $\Delta$. Пусть $e_{1}$ вершина из $\left(\left[c_{1}\right] \cap\left[d_{1}\right] \cap[a]\right)-\Delta$. По условию 3$)$ теоремы в точности $\alpha$ вершин из $\mu$-подграфа $[b] \cap\left[e_{1}\right]$ содержится в $[a]$. Поскольку $n \geqslant 6$, то подграфы $A$ и $B$ содержат не менее $\alpha$ вершин каждый. Так как $c_{1} \in A, d_{1} \in B$ и $\left\{c_{1}, d_{1}\right\} \subset\left(\left[c_{1}\right] \cap\left[d_{1}\right] \cap[a]\right)-\Delta$, то вершина $e_{1}$ не смежна с некоторой вершиной $f_{1}$ из $A$ и с некоторой вершиной $g_{1}$ из $B$. С другой стороны, по выбору вершины $a$ разность $(A \cup B)-\left[e_{1}\right]$ является кликой. Значит, $(A \cup B)-\left[e_{1}\right]-$ клика, содержащая ребро $f_{1} g_{1}$. Поскольку $A \cup B$ является $\beta$-расширением $((n-2) \times 2)$-графа, то $(A \cup B)-\left[e_{1}\right]$ содержит не более $2 \beta$ вершин. Но тогда $\left|(A \cup B) \cap\left[e_{1}\right]\right|$ ограничено снизу числом $6 \beta>\alpha$. Противоречие с условием 3$)$ теоремы для $\mu$-подграфа $[b] \cap\left[e_{1}\right]$.

Пусть $[b]$ является $\beta$-расширением графа Шлефли при $\beta=\alpha / 2$. В этом случае $\left|K_{\Delta}(a)\right|=\beta$ и подграф $([a] \cap \Delta)-K_{\Delta}(a)$ будет $\beta$-расширением граффа Клебша с параметрами $(16,10,6,6)$ (см., например, $[8$, п. 10.3.3]). Этот граф изоморфен графу на бинарных четного веса векторах размерности 5. Два вектора смежны тогда и только тогда, когда расстояние Хэмминга между ними равно 2 . Пусть $c_{1}, d_{1}$ являются несмежными вершинами $([a] \cap \Delta)-K_{\Delta}(a)$. По условию 3$)$ теоремы в точности $\alpha$ вершин из $\mu$-подграфа $\left[c_{1}\right] \cap\left[d_{1}\right]$ содержится в $[a]$. В точности $\beta$ из них принадлежит $K_{\Delta}(a)$. В то же время подграф $\left(\left[c_{1}\right] \cap\left[d_{1}\right] \cap a^{\perp}\right)-K_{\Delta}(a)$ содержит в точности $6 \beta$ вершин. Таким образом, $\beta$ вершин из $\left[c_{1}\right] \cap\left[d_{1}\right] \cap a^{\perp}$ не принадлежат $\Delta$. Пусть $e_{1}$ вершина из $\left(\left[c_{1}\right] \cap\left[d_{1}\right] \cap[a]\right)-\Delta$. По условию 3$)$ теоремы в точности $\alpha$ вершин из $\mu$-подграфа $[b] \cap\left[e_{1}\right]$ содержится в $[a]$. 
Значит, подграф $([a] \cap \Delta)-K_{\Delta}(a)$ содержит не более чем $\alpha$ вершин из $\left[e_{1}\right]$ и не менее $\alpha$ вершин вне $\left[e_{1}\right]$. Однако $\beta$-расширение графа Клебша не содержит клик из $\alpha$ вершин. Таким образом, среди вершин из $([a] \cap \Delta)-K_{\Delta}(a)$ не смежных с $e_{1}$ есть пара несмежных вершин. Противоречие с выбором вершины $a$. Лемма доказана.

Завершим теперь доказательство теоремы 2.

По леммам 3.1 и 3.2 окрестность любой вершины из Г является графом типа (1), (2), (3) из заключения леммы 3.1. Заметим, что если $a$ и $b$ смежные вершины из $\Gamma$, то подграф $[a] \cap[b]$ является одновременно окрестностью вершины $a$ в $[b]$ и окрестностью вершины $b$ в $[a]$. Таким образом, из связности графа $Г$ и строения графов, перечисленных в заключении леммы 3.1 , следует, что все окрестности в $Г$ изоморфны графу $G$, которьй является одним из следующих графов:

(1) $\beta$-расширение $(m \times n)$-графа при $\beta=\alpha / 2$ и $m \geqslant 3, n \geqslant 3$;

(2) $\beta$-расширение треугольного графа $T(n)$ при $\beta=\alpha / 4, n \geqslant 6$;

(3) $\beta$-расширение графа Шлефли при $\beta=\alpha / 8$.

Для доказательства теоремы нам теперь достаточно показать, что в последних двух случаях $\beta=1$. Заметим, что окрестность вершины в любом $\mu$-подграфе из $Г$ является $\mu$-подграфом в окрестности этой вершины в графе $\Gamma$.

Пусть имеет место случай (2).

Любой $\mu$-подграф $\beta$-расширения треугольного графа $T(n)$ является $\beta$-расширением $K_{2,2}$. Значит, если $\Sigma$ является $\mu$-подграфом из $\Gamma$, то $\Sigma$ - локально $\beta$-расширение $K_{2,2^{-}}$ графа и в то же время подграф из $G$. В частности, $\Sigma$ является реберно регулярным графом без 3-лап.

По теореме 1 из [2] реберно регулярньй граф диаметра 2 без 3-лап является одним из следуюших графов:

(a) $K_{t \times 2}$ при $t>1$;

(b) дополнение для сильно регулярного графа с параметрами $(v, k, 0, \mu)$ при $\mu<k$;

(c) $(n \times n)$-граф при $n \geqslant 3$;

(d) треугольньй граф $T(n)$ при $n \geqslant 5$;

(е) граф Шлефли;

(f) пятиугольньй граф $P(t)$ при $t \geqslant 2$ с числом вершин $5 t^{2}$ (см. [2]).

В этом списке локально $\beta$-расширением $K_{2,2}$-графа является только граф из пункта (a) при $t=3$. Отсюда, если каждая связная компонента из $\Sigma$ имеет диаметр 2 , то $\Sigma$ изоморфен либо $T(4)$, либо объединению изолированных подграфов, изоморфных $T(4)$. В частности, $\beta=1$.

По теореме 2 из [2] если реберно регулярный граф диаметра больше 2 без 3-лап, то он либо является граффом икосаэдра, либо все его $\mu$-подграфы имеют не более двух вершин. Так как $\Sigma$ - локально $\beta$-расширение $K_{2,2}$-графа, то $\Sigma$ не может иметь связных компонент диаметра больше 2 .

Пусть имеет место случай (3).

Любой $\mu$-подграф $\beta$-расширения графа Шлефли является $\beta$-расширением $K_{4 \times 2}$. Значит, если $\Sigma$ является $\mu$-подграфом из $\Gamma$, то $\Sigma$ локально $\beta$-расширение $K_{4 \times 2}$-графа. В частности, $\Sigma$ является реберно регулярньм графом без 3 -лап. Применим теоремы 1 и 2 из [2] к связной компоненте $X$ из $\Sigma$. Получаем, что $X$ изоморфна $K_{5 \times 2}$. Значит, $\beta=1$ и в этом случае.

Теорема 2 доказана. 
4. Доказательство теоремы 3. Пусть граф Г удовлетворяет условию теоремы 3 и все $\mu$-подграфы из $Г$ имеют диаметр 2. Тогда по теореме 2 граф $\Gamma$ локально $G$-граф, где $G$ является одним из следуюших графов:

(a) $\beta$-расширением $(m \times n)$-графо при $\beta=\alpha / 2$ и $m \geqslant 3, n \geqslant 3$;

(b) треугольньг графом $T(n)$ при $n \geqslant 6$;

(c) графом Шлефли.

В силу результата Нуматы [3] для доказательства теоремы 3 нам достаточно показать, что если граф Г удовлетворяет условию теоремы 2 и все $\mu$-подграфы из $Г$ диаметра 2 , то все $\mu$-подграфы из $\Gamma$ - изоморфные реберно регулярные графы.

Пусть $\Sigma$ является $\mu$-подграфом из Г. Ввиду того, что теорема 2 определяет локальное строение графа $\Gamma$, мы имеем также локальное строение для графа $\Sigma$.

Пусть, например, Г является локально $G$-графом, где $G-\beta$-расширение $(m \times n)$-графа при $\beta=\alpha / 2$ и $m \geqslant 3, n \geqslant 3$. В этом случае окрестность любой вершины из $\Sigma$ будет объединением двух изолированных $\beta$-клик, т.е. $\Sigma$ является реберно регулярным графом диаметра 2. Но тогда по теореме 1 из [2] $\Sigma$ - либо $((\beta+1) \times(\beta+1))$-граф, либо пятиугольньй граф $P(t)$ при $2 t=\beta+1$. Однако в последнем случае граф $\Sigma$ содержит цикл длины 5 . Так как $\Sigma$ является $\mu$-подграфом из $\Gamma$, то $\Sigma$ - подграф из окрестности некоторой вершины графа $\Gamma$. Заметим, что ребро из $\Sigma$ не может содержатся в $\beta$-клике ядра вершины из этой окрестности в силу локального строения $\Sigma$. Но $(m \times n)$-граф не содержит циклов длины 5. Таким образом, пятиугольный граф $P(t)$ не может быть $\mu$-подграфом из Г. Значит, все $\mu$-подграфы из Г изоморфные реберно регулярные графы диаметра 2. Аналогично рассматриваются остальные случаи. Теперь утверждение теоремы 3 следует из теоремы Нуматы [3].

\section{СПИСОК ЦИТИРОВАННОЙ ЛИТЕРАТУРЫ}

[1] Brouwer A.E., Numata M. A characterization of some graphs which do not contain 3-claws // Discrete Math. 1994. V. 124. P. 49-54.

[2] Numata M. On a characterization of a class of the regular graphs of diameter 2 // Osaka J. Math. 1974. V. 11. P. 389-400.

[3] Numata M. A characterization of Grassmann and Johnson graphs // J. Combin. Theory. Ser. B. 1990. V. 48. P. 178-190.

[4] Кабанов В. В., Махнев А. А. Кореберно-регулярные графы без 3-лап // Матем. заметки. 1996. Т. 60. № 4. С. 495-503.

[5] Кабанов В. В., Махнев А. А. Об отделимых графах с некоторьми условиями регулярности // Матем. сб. 1996. Т. 187. №10. С. 73-86.

[6] Кабанов В.В., Махнев А.А. Графы без 3-лап с равномошными $\mu$-подграфами // Изв. Урал. гос. ун-та. Матем. и мех. Вып. 1. 1998. № 10. С. 44-68.

[7] Махнев А. А. Об одном классе граффов без 3-лап // Матем. заметки. 1998. Т. 63. № 3. C. $407-413$.

[8] Brouwer A.E., Cohen A.M., Neumaier A. Distance-Regular Graphs. Berlin: Springer-Verlag, 1989.

[9] Blokhuis A., Brouwer A. E. On Locally 4-by-4 Grid Graphs // J. Graph Theory. 1989. V. 13. № 2. P. 229-244.

[10] Hall J. I. Classifying copolar spaces and graphs // Quart. J. Math. 1982. V. 33. P. 421-449.

[11] Neumaier A. Characterization of a class of distance regular graphs // J. Reine Angew. Math. 1985. V. 387. P. 182-192. 